\title{
POWER SPECTRAL STUDIES OF VHF SCINTILLATIONS OVER NEAR EQUATORIAL STATION ANANTAPUR
}

\author{
Dr. B. Rama Murthy', Dr. U. Eranna', Dr. K. Bhanu Prasad ${ }^{3}$, R.Manjula $^{4}$ \\ ${ }^{1}$ Department of Instrumentation, Sri Krishnadevaraya University, Anantapur-515003, \\ ${ }^{2,3,4}$ Department of Electronics and Communication Engineering, Bellary Engineering College, \\ E-mail: ${ }^{1}$ ramamurthy_ugc@yahoo.com, ${ }^{2}$ jayaveer_88@yahoo.com, \\ ${ }^{3}$ bhanu1955@37.com, ${ }^{4}$ rajamanjula@rediffmail.com
}

\begin{abstract}
The invest igat ions on lonosphere developed largely because of the ionospheric communicat ions, the improvement of which depends upon the increased knowledge of the ionospheric behavior and it s characterist ics. The study of radio wave scint illat ions plays an important role in understanding the basic mechanisms of the generat ions of ionospheric irregularit ies as well as planning of atellite based communicat ion systems and developing satellite based radar systems. In this paper we present the digitally recorded ionospheric scint illat ion data of $150.649 \mathrm{MHz}$ signal from FLEETSAT satellite at near equatorial station Anantapur, acquired through a PC based real t ime data acquisit ion system and analyze this data to evaluate the $S 4$ index, the auto correlat ion function and power spectra.
\end{abstract}

Key words: Ionosphere, Power Spectral, Scint illations, Scintillation Index.

\section{INTRODUCTION}

When a radio wave traverses through the ionosphere containing free electronics, a phase change occurs along the same path. In addition, the ionosphere contains irregularities f plasma density, phase fluctuations build-up across the wave front. As the wave propagates away from the onosphere, phase mixing occurs and fluctuations in amplitude are developed (Amplitude scintillations). The fluctuations in amplitude, phase as well as angle-of-arrival of scintillations have been observed at frequencies ranging from $10 \mathrm{MHz}$ to several $\mathrm{GHz}$. By studying these fluctuations, much information about the characteristics of the ionosphere can be understood $[1,11,25,30]$. Early observations were made mostly at single frequencies. It is well known that the departure of the relative dielectric permittivity from its background value in the ionosphere is inversely proportional to the square of the signal frequency. Therefore, under these conditions for which fluctuations occur at higher frequencies, signals at lower frequencies will suffer much more severe scintillations. In fact, at times the scintillation will be so intense that the multiple scattering effects will become important [33]. The Radio Beacon Experiments on ATS-6, with the three frequencies at 40, 140 and $360 \mathrm{MHz}$, provided a good opportunity for simultaneous multi frequency observation of the scintillation phenomenon. Results of the ATS 6 scintillation data are reviewed and the results of the analysis of the FLEETSAT scintillation data are also presented in this paper.

\section{WEAK AND STRONG SCINTILLATIONS}

\section{A Weak Scintillation}

For the analytical study of the scintillation phenomena, it is necessary to solve the inhomogeneous wave equation presented below, for the electric field $\mathrm{E}$.
$V^{2} E+k^{2} \varepsilon(r) E=-V[E . V 1 n \varepsilon(r)]$

The permittivity $\varepsilon(r)$ is a random function of position $r$, and $k$ is the wave number ( $k=2 \pi / \lambda, \lambda$ is the wavelength). First order perturbation techniques provide solutions in the limit of single scatter. For an incident plane wave

$E_{0}(r)=E_{0} \exp (-i k . r)$

Where $E(r)$ can be written as

$E(r)=E_{0}(r) \exp [\Psi(r)]$

Where

$\Psi(r)=r_{e} ? N_{e}\left(r^{\prime}\right) \exp \left[i k .\left(r-r^{\prime}\right)\right] \exp \left(i k\left|r-r^{\prime}\right|\right) d r^{\prime} / r-r^{\prime} \mid$

Where re is classical electron radius, $\Delta \mathrm{Ne}\left(r^{\prime}\right)$ is electron density fluctuation (departure from mean) at $r$ '. The Scintillation Index S4 is a very important parameter in characterizing the scintillations. It is the normalized variance (second moment) of the intensity of the wave, and is expressed in terms of the intensity or the amplitude of the wave as

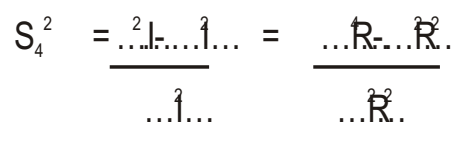

I is the intensity of the wave. Since I = R2 where R - is the amplitude of the wave.

Further, since

$\Psi(r)=\chi(r)+i s(r)$

then for weak scintillation such that . .???t can be shown [23] S4 $2=4<\wedge 2$. Then for

single scattering, $[17,32]$ showed 


$$
\begin{aligned}
& \left|\chi^{2}\right|=\frac{\pi^{2} \mathrm{~K}^{2} \mathrm{~L}}{2}{\frac{\mathrm{f}_{\mathrm{p}}}{\mathrm{f}_{\mathrm{t}}}}^{4} \quad \mathrm{~F}_{\mathrm{F}}(\mathrm{k}) \mathrm{Q}_{\mathrm{N}}(\mathrm{K}) \mathrm{KdK} \\
& \mathrm{F}_{\mathrm{F}}(\mathrm{K})=1-\frac{2 \mathrm{~K}}{\mathrm{k} 2(\mathrm{~L})} \sin \left[\frac{\mathrm{k}^{2} \mathrm{~L}}{2 \mathrm{~K}}\right] \cos \left[\frac{\mathrm{k}^{2}}{\mathrm{~K}}(\mathrm{Z}-\mathrm{L} / 2)\right]
\end{aligned}
$$

$F F(K)$ is Fresnel filtering function, $L$ is thickness of the irregularity layer, $Z$ is distance to the top of the irregularity slab, $k$ is spatial wave number, $\Phi N(K)$ is the power spectrum of the irregularities, $\mathrm{ft}$ is the transmitted frequency and $\mathrm{fP}$ plasma frequency of the background ionosphere. And Fresnel Zone Size

$$
\operatorname{Rf}(\text { Radius })=\sqrt{\lambda}(2 Z-L)
$$

Fresnel Frequency $\mathrm{ff}=\mathrm{V} / \sqrt{\lambda}(2 \mathrm{Z}-\mathrm{L})$

Where $V=$ is the velocity of the irregularity. Measuring Fresnel frequency and assuming the height $Z$ can estimate the velocity of the irregularity estimated from equations 8 and 9 .

\section{B. Strong Scintillation}

As it has been observed that, the single scatter problem has been solved and though some approximations have been made, the results can still be compared quantitatively with the experimental data. In sharp contrast, the multiple scatter problems are so complex that in simplifying it to a level at which it can be handled, the results can only be compared semi-quantitatively with the experimental observations. [17,33] have done theoretical computations which have shown that under multiple scatter theory, it is no longer possible to obtain a constant exponent ' $n$ ' that describes the frequency dependence of the scintillation index S4 as indicated in equation 4 . Rather, the frequency dependence is a function of the signal frequency and other parameters of the irregularity slab. Also, under certain conditions, the scintillation index can be greater than unity. This is referred to as focusing [33]. This phenomena occurs at a distance zfoc from the center of a thin irregularity slab [29] where

$$
S 4 \propto-n=f(P+2) / 4 \hat{A} \hat{A}
$$

Therefore

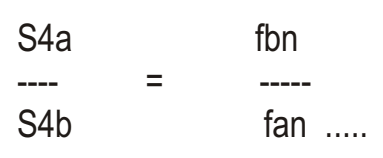

Where $n=(p+2) / 4$ and is found to be about 1.5 for strong scintillation and fa and fb are the transmitting frequencies.
Zfoc a ro $2 \mathrm{k} /$ Фo...

Фo $2=\mathrm{K} 2(\mathrm{fp} / \mathrm{ft}) 4 \mathrm{roL}<(\Delta \mathrm{N} / \mathrm{No}) 2^{\wedge} / 4 \hat{\mathrm{A}}$. ..

$[19,27]$ have shown that under strong scint illat ion, the power spectrum will be broadened at the low-frequency-end while the slope of the roll off will remain the same as under single scattering. They also found that the correlat ion distance or the autocorrelat ion interval ( $t$ ime $\mathrm{Tm}$ required for the autocorrelat ion coefficient to decrease to 0.5 ) would decrease with increasing transmitted frequency under weak scint illat ion but would increase with mult iple scatter under strong scint illat ion.

\section{THEORY OF POWER SPECTRA AND SCINTILLATION INDICES SI AND S4}

\section{A. Scintillation Index (SI)}

The relation represents the Scintillation Index

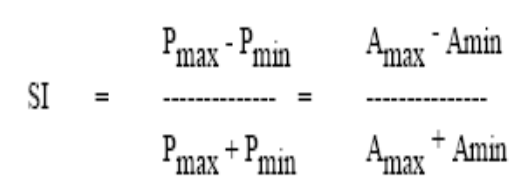

Where $\mathrm{SI}$ is the Scintillation Index gives the extent/measure of large range of scale sizes of rregularities, Pmax is the power amplitude of the third peak down wards the maximum

excursion, Pmin is the power amplitude of the third trough up wards the minimum excursion and $A$ is amplitude level up from the minimum excursion. The recorded scintillation measurements are scaled every 15 minutes and SI values for each sample record are estimated and the mean scintillation index SI of the entire record is estimated.

\section{B S $\Phi$}

The hourly occurrences of scintillation data were plotted to locate the periods of scintillation. It was found that this phenomenon was most likely to occur between 2100 and 0300 hrs local time. A large number of segments of data of sufficiently long duration, of at least 15 minutes and above only were chosen for the present analysis. To obtain a measure of the intensity of the fading and its statistics, the scintillation index S4 has been computed from equation 7 where the intensity (I) was substituted by the square of the amplitude (R) of the received signal. Each data segment was sub-divided into blocks of about 512 points and S4 was computed for each block. Then the average value S4 was computed for the entire 
scintillation record. By verifying that the scintillation index was relatively constant throughout the entire period being considered for the present analyses and it was supplemented by additional assurance that the process was stationary. It is an established fact that the computed power spectra are sensitive to large low frequency trends. Hence, all data were passed/filtered through a weighted running mean high pass digital filter with a cut off frequency of $0.005 \mathrm{~Hz}$. The detrended data have almost zero mean and are $180^{\circ}$ out of phase with the input. Also due to the transient response of the filter, a number of data points equal to the number of filter coefficients had to be discarded (half from the beginning and half from the end of the data segment). With the detrended data, S4 is calculated. However, before doing so, the $180^{\circ}$ phase shift had to be removed, and the mean is restored to be close to zero. This was necessitated because the inversion of the data obviously changes its features, and since the scintillation index is essentially the

normalized variance of the intensity fluctuations, the mean is also a very important quantity. As a final check for stationary, the criteria adopted for a long period of data ( ${ }^{\wedge} 45$ $\min$.) the standard deviation of S4 for the various blocks should be less than $20 \%$. For shorter segments ( $15 \mathrm{~min}$.) a standard deviation of only $10 \%$ is tolerated.

For the recent years, the use of the power spectrum of temporal amplitude fluctuations being used for the study of the ionospheric irregularities has gained recognition. There are many ways in which this parameter may be computed. [10] Chose

to utilize the fact that the power spectrum is the Fourier transform of the auto-correlation function. This proved to be a good choice. Further, behavior of the auto-correlation function itself is also of interest in determining certain characteristics of the scintillations. Due to the fact that data segments do not extend to plus and minus infinity, the resulting spectra will only be an estimate of the true spectra [5] further, the autocorrelation function can only be computed for a finite number of lags. By truncating this function, we are, in effect, multiplying it by a "window function". Since a multiplication in time is a convolution in frequency, our estimated spectra will also be convolved with the Fourier transform of the window function. If the autocorrelation function were merely truncated, a plain rectangular widow would effectively have been used. This window has $\sin (w / 2)$ / (w/2), type of frequency response that exhibits very large side-lobes and for this reason is undesirable. Hence, the Hanning window, which exhibits very small side-lobes, was used, though; it has a wider main lobe and is represented by

$W(t)=0.5+0.5 \cos (\pi t / T m)$
' $\mathrm{Tm}$ ' is the length of the auto-correlation function or the autocorrelation interval in seconds. (i.e. the time required for the autocorrection function to decrease to 0.5 ). The presence of the side-lobes makes it necessary, that the mean (zero frequency or D.C. component) and any large low frequency components be removed from the data. If the mean or any low frequency trends are large with respect to the fluctuations, the contribution in power from the side lobes during the convolution would also be largeand thus distort the real power spectrum. The width of the window in the time domain, or specifically, the number of lags in the autocorrelation function, will determine the width of the major lobe in the frequency domain. A wider window in time will produce a narrower window in frequency and thus allow frequency components which are closer together to be resolved instead of being smoothened or averaged together. At the same time however, it must be realized that computing an increased number of lags for the same length of data will decrease the number of degrees of freedom and hence decreases the stability or reliability of the spectrum[18].

$\mathrm{Kf}=2.5(\mathrm{~T} / \mathrm{Tm})$

Where ' $T$ ' is the length of the data segment and $\mathrm{Tm}$ is the auto-correlation interval in seconds. The frequency resolution (full width e-1) is given by

$\Delta \mathrm{fr}=1 / \mathrm{Tm}$

Thus it can be seen that there is going to be a trade off between the frequency resolution and the stability of the spectra. According to [33] a resolution of $0.005 \mathrm{~Hz}$ was necessary, and hence about 200 lags were computed from all cases. The degrees of freedom varied for different data sets, but were adequate in all cases. At this point, the power spectrum of the scintillation record is obtained by using the Fast Fourier Transform algorithm. Since this is done digitally, the power spectrum is only evaluated at discrete frequencies, the resolution between them being given as

$\Delta \mathrm{f}^{\prime}=1 / \mathrm{NT}^{\prime}$

$\mathrm{N}$ is the number of points being transformed and $\mathrm{T}^{\prime}$ is the period in seconds between samples. Since the behavior between these discrete frequencies is unknown, it is desirable to space the frequencies rather closely to avoid what is known as the "picket-fence-effect" [20]. This can be done without altering the data by merely adding zeros to the end of the auto-correlation function. In effect, its length has been increased ten fold without altering its content, and we are thus enabled to interpolate between what would otherwise be widely spaced frequencies. 


\section{DATA PREPARATION}

In the present study an attempt has been made by the author to compute a Fourier power spectrum, we first filter the data to remove any large low frequency trends and then subtract out the mean. The autocorrelation function is computed for 256 lags, multiplied by the Hanning window, added zeros at the end, and finally processed through the FFT.

The resulting power spectrum must be compensated for the previously mentioned post detection filters in the receiver. To do this, it is realized that the analog filter has a frequency response whose magnitude is given by $(\omega 2+$ $1)-1 / 2$, and the averaging has a frequency response of 2 $\sin (\omega 2) /(\omega / 2)$. The total filtering effect is the product of the two magnitudes, and to compensate for its effect, the power spectrum has to be multiplied by its inverse. The performance of this operation will put the power spectrum close to its proper perspective, but the effects of the filtering are irreversible. Therefore spectral features above about $0.1 \mathrm{~Hz}$ should be viewed with caution when used with very low frequency filters with cut-off frequency $\$ 0.1$. The data are considered fit for the computation of the Bessel spectrum. Due to the presence of large number of low frequency components in the auto-correlation function, it was found to be necessary to use a "nrowhitonod_v/arcinn" of the Reccol transform (Lovelace $\mathrm{f}_{\mathrm{B}}(\mathrm{f})=\begin{array}{ccc}1 & 2 & \mathrm{dA}(\tau) \\ --- & \tau J_{1}(\Omega \tau) & -\cdots \\ 2 \pi & d \tau\end{array}$

$A(T)$ is the autocorrelation function. The problems associated with this transformation are similar to those encountered with the Fourier spectrum. A Gaussian lag window

$[(\exp (\mathrm{Y}(\mathrm{T} / \mathrm{Tm}) 2)]$ with $\mathrm{g}=4$ has to be used to suppress sidelobes of the frequency response. According to [18] the frequency resolution (full width e-1) and the degrees of freedom are established by

$\Delta \mathrm{fB}=\mathrm{\gamma} 1 / 2 / \mathrm{Tm} \ldots$

$\mathrm{KB}=2(\mathrm{~T} / \mathrm{Tm})(\mathrm{\gamma} / \mathrm{ftm}) \ldots$

The 'pickett-fence-effect' is also a problem which case is handled by evaluating the integral at very closely spaced frequencies of the order of $0.0005 \mathrm{~Hz}$. To compute the Bessel spectrum, the Gaussian lag window multiplied the differentiated auto-correlation function, and the resulting integrand was integrated using Simpson's rule.

Before plotting, the pre-whitening was removed. The filter distortion, however, was not compensated and in this case due to the fact that the higher frequencies are highly unstable anyway.

\section{DATA ACQUISITION AND METHOD OF POWER SPECTRALANALYSIS}

In the existing Real time data acquisition system the output of the ICOM receiver is passed through a low pass filter with a high frequency cut-off approximately fixed at 1 $\mathrm{Hz}$ to suit the response speed of the pen (strip chart recorder not used in the present study). In doing so, all the high frequency components in the scintillation signal are removed. Hence, this signal will not yield true power spectrum when subjected to FFT analysis. In order to overcome this difficulty, additional hardware modifications are incorporated outside the receiving system. The receiver output is applied simultaneously to two buffer amplifiers designed with LM 324. This reduces the loading on the receiver when an additional digital recording channel is incorporated. The output of the second buffer amplifier is connected to another low-pass filter designed with a high frequency cut-off at $1 \mathrm{KHz}$, serves as a digital data acquisition channel. The output of this second filter is applied to an Analog to Digital converter and to the personal computer through suitable interface cards. The scintillation signal data are being sampled at with an $A / D$ converter ICL 7109 at 16 samples/second for few samples and for certain chosen occasions a fast $A / D$ converter AD 1674 with a sampling rate of 256 samples/sec is employed which allows for the detection of all possible high frequency components in the scintillating signal.

\section{OPTIMUM LENGTH OF THE SAMPLE RECORD OF SCINTILLATIONS AND CATEGORIZATION}

The scintillation data are recorded at Anantapur using 250.649 MHz VHF signals transmitted from FLEETSAT satellite continuously during the period March 2005 to August 2006. For the present study certain typical scintillation data records are selected for the power spectral analysis, covering the three seasons namely Equinox, winter and summer. Further, they include weak and strong scintillations as well as they are active during Premidnight and Post-midnight periods. Majority of these scintillation events are recorded with the help of high speed A/D converter AD 1674 with sampling rate of 256 samples/second, and few records recorded 
Table 1. Details of Scintillation Records Selected for Power Spectral Studies and Results of the Analysis. * Lunar Eclipse Preceding day, ${ }^{* *}$ Large Sample Record of 22440 Data Points

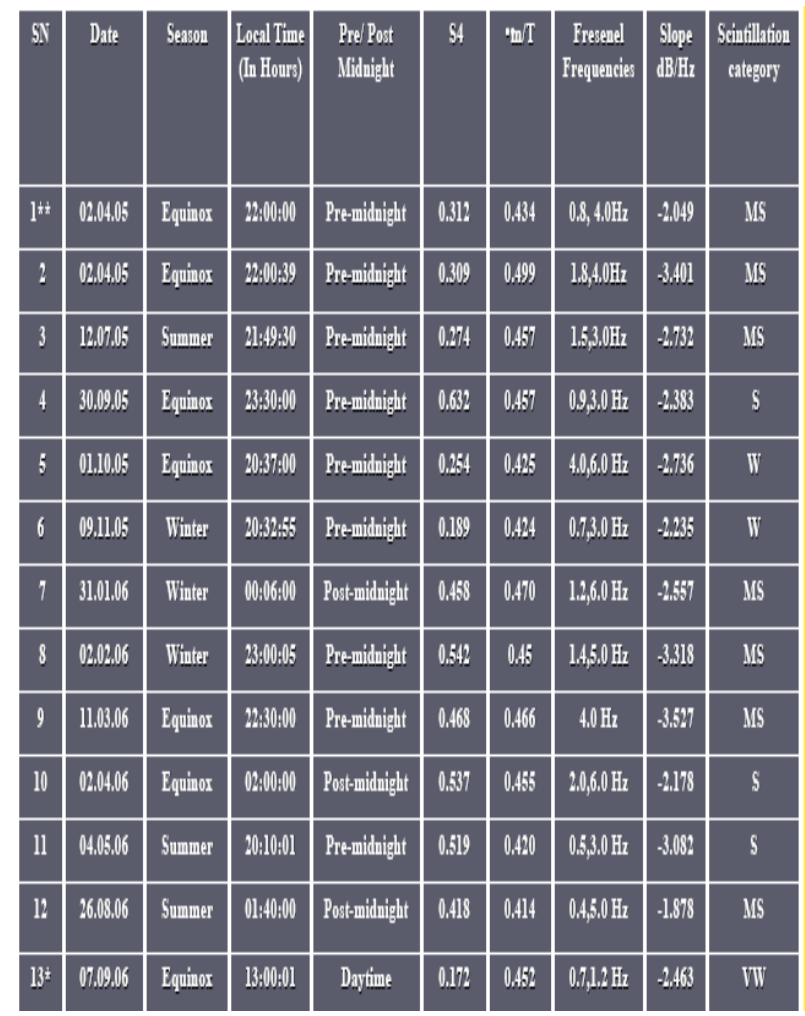

\section{A. Studies on a Large Sample record of Scintillations}

A typical record of VHF scintillations observed on 2nd April 05 from 2200 to 2234 hrs LT (34 minutes duration) is considered in the present study. The scintillation record (Raw Data of Amplitude Scintillations) is presented in Fig. $1(A)$. The scintillating signals are sampled with an A/D converter of moderate speed of 11 samples/second. Hence, a total number of 22440 data points are sampled during these 34 minutes of recording. These data are subjected to Fast-Fourier- Transform technique for obtaining the power spectrum. The resulting phase spectrum and power spectrum of these scintillations are presented in Fig. $1(B, C)$ respectively. The spectral power is expressed in arbitrary units in $\mathrm{dB}$. It can be seen from these figures $1(B, C)$ that, though the major trends are projected distinctly, the minor features are suppressed in projecting the statistical average trends over a large length of the data. Further, a large number of periodicities present in the raw data are smoothened out in the spectrum of a large sample. The auto-correlation function is presented in Fig. $1 \mathrm{D}$. The auto-correlation interval (the time required for the autocorrelation coefficient to fall from 1.0 to 0.5 ). ' $\mathrm{Tm}$ ' is found to be 512.6 seconds and the total time required for the correlation coefficient to fall from 1.0 to zero ' $T$ ' is found to be 1046.12 seconds. Hence, the ratio $\mathrm{Tm} / \mathrm{T}$ is found to be 0.49 .

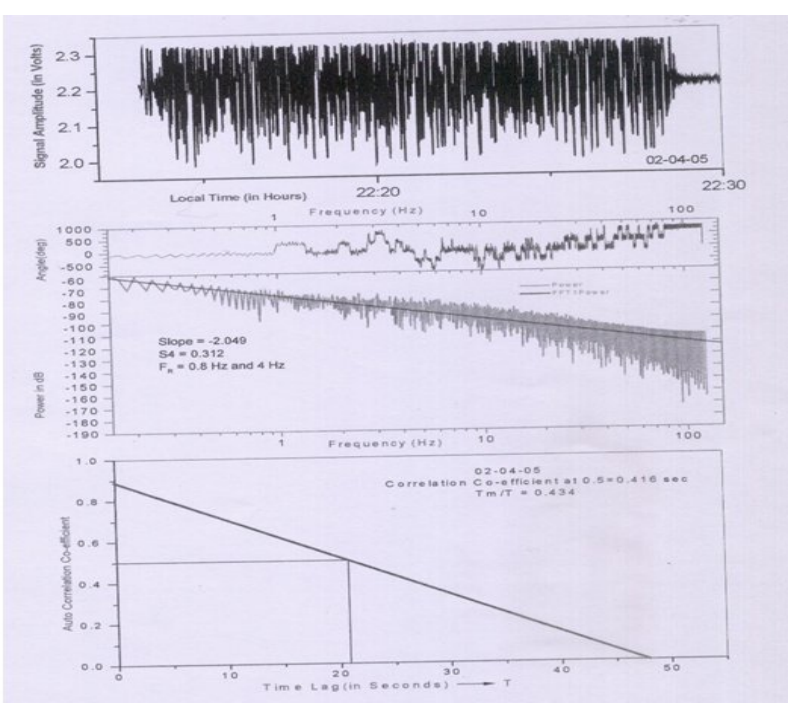

Fig. 1. Power spectral characteristics of scintillation recorded on 2nd April 06 (large sample (A) Raw Data, (B)

Phase Spectrum, (C) Power Spectrum and (D) Autocorrelation.

\section{B. Studies on a Small Sample of Scintillations}

Here, in order to bring out the minor details more distinctly, the large record has been split into a number of smaller records with sample lengths of 512 data points (twice the number of data points sampled by the high speed $A / D$ converter). The short scintillation record (Raw Data of Amplitude Scintillations) is presented in Fig. 2 (A). This record is also subjected to Fast-Fourier-Transform technique for obtaining the power spectrum as in the previous case. The resulting phase spectrum and power spectrum are presented in Fig.2 $(B, C)$ respectively. The spectral power is expressed in arbitrary units in $\mathrm{dB}$.

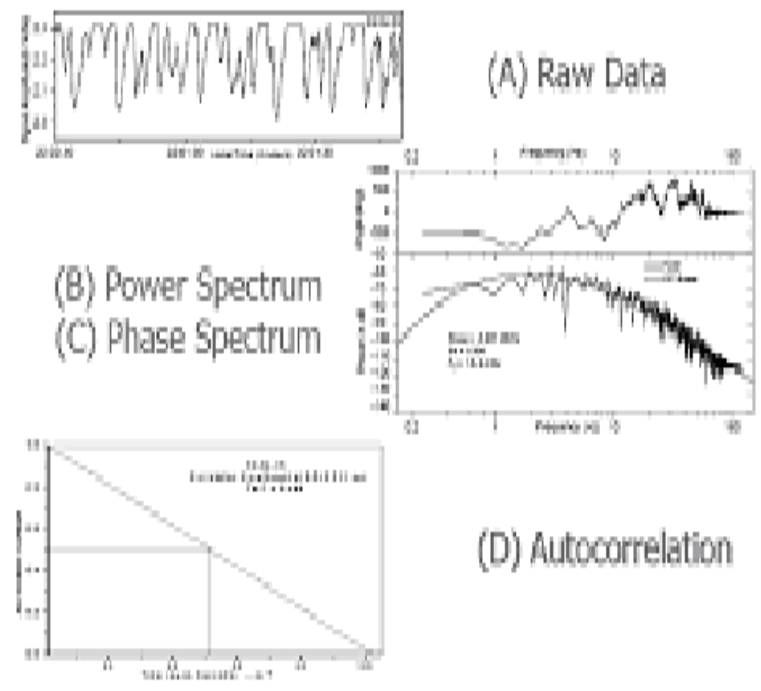

Fig. 2. Power spectral characteristics of scintillation recorded on 2nd April 05 (A) Raw Data, (B) Phase Spectrum, (C) Power Spectrum and (D) Autocorrelation 
It can be seen from these two figures that a large number of minor features like the number of periodicities present in the raw data which are suppressed in the analysis of a large record are projected distinctly, while retaining the general trends without any changes.A close examination of the results of the analysis of the large record presented in Table 7.1 such as, the spectral indices S4, the Fresnel frequencies and the asymptotic slopes are in good agreement with those obtained from the analysis of the smaller records.

Further, an examination of the Fig. 2 (D), the "auto correlation interval" (the time required for the autocorrelation coefficient to fall from 1.0 to 0.5 ) $\mathrm{Tm}$ is found to be 0.51 seconds while the total time required for the correlation coefficient to fall from 1.0 to zero ' $T$ ' is found to be 1.02 seconds. This results in a $\mathrm{Tm} / \mathrm{T}$ ratio of 0.499 that is in close agreement with that for the large record. This also confirms that by reducing the length of the sample does not affect the results but brings out the minor characteristic more distinctly,while retaining the broad features unaffected. Hence, smaller samples of 512 data points are only considered for the analysis of the remaining scintillation records.

\section{Categorization of the Scintillations}

The scintillations can be categorized depending upon their intensities. The spectral indices SI and S4 are normally considered as the measures of the intensities of the scintillation.

Table 2. Categorization of Scintillations on the basis of $\mathbf{S} 4$ Index

\begin{tabular}{|c|c|c|}
\hline S $_{4}$ Index & $\begin{array}{c}\text { Scintillatio } \\
\text { n category }\end{array}$ & $\begin{array}{c}\text { Nomenclat } \\
\text { ure } \\
\text { adopted }\end{array}$ \\
\hline 0.10 & $\begin{array}{c}\text { No } \\
\text { Scintillatio } \\
\mathrm{n}\end{array}$ & NS \\
\hline $\begin{array}{c}>0.10 \text { to } \\
0.20\end{array}$ & Very Weak & VW \\
\hline $\begin{array}{c}>0.20 \text { to } \\
0.25\end{array}$ & Weak & W \\
\hline $\begin{array}{c}0.25 \text { to } \\
0.50\end{array}$ & $\begin{array}{c}\text { Moderately } \\
\text { Strong }\end{array}$ & MS \\
\hline $\begin{array}{c}0.50 \text { to } \\
0.75\end{array}$ & Strong & S \\
\hline$>0.75$ & $\begin{array}{c}\text { Very } \\
\text { Strong }\end{array}$ & VS \\
\hline
\end{tabular}

Higher values of SI and S4 indicate the higher intensities and lower values indicate lower intensities. While SI indicates the extent of the range of scale sizes irregularities, S4 indicates the scintillations suffered by the radio wave. Hence, S4 represents more scientifically about the strength or the intensity of the scintillations than SI. Hence, the categorization is normally done based on S4 as shown in Table 2. Further, the ratio of the auto-correlation interval $\mathrm{Tm}$ (time required for the autocorrelation coefficient to fall to 0.5 ) and $T$ (the total time period required for the autocorrelation coefficient to fall to zero) i.e. ( $\mathrm{Tm} / \mathrm{T}$ ) is also taken as an index of intensity of the scintillation. For strong scintillation $\mathrm{Tm}$ falls more rapidly than for weak scintillation for which it takes longer time. Hence, smaller values for the ratio indicate stronger scintillations while larger values indicate weaker scintillations. Additionally, the slope of the asymptote at the high frequency-end of the scintillation power spectrum is also considered as an index of the strength (intensity) of the scintillation. The intensity is proportional to the magnitude of the slope.

\section{RESULTS OF POWERSPECTRAL STUDIES OF SOME TYPICAL SCINTILLATION RECORDS}

\section{A. Power Spectral Studies on the Scintillation Record of 12th Ouly $255 \mathrm{G}$}

This scintillation event recorded during summer period recorded with a slow speed A/D converter with eleven samples/second. Further, this is a postmidnight event with S4 $=0.383$. Hence, it is characterized as a moderately strong scintillation. The scintillation record is presented in Fig. $3(\mathrm{~A})$.

The resulting phase spectrum and power spectrum are presented in Fig. $3(B, C)$ respectively. The spectral power is expressed in arbitrary units in $\mathrm{dB}$. The "autocorrelation" function is presented in Fig.3 (D).

The power spectrum shown in Fig.3 (B) exhibits a flattening at the low-frequencyend followed by a moderately steeper roll-off $(-2.73 \mathrm{~dB} / \mathrm{Hz})$ at the high frequencies with asymptotic breaks around $1.5 \mathrm{~Hz}$ and around $3.0 \mathrm{~Hz}$. These break points are known as Fresnel-Frequencies [8,14,21]. The magnitude of broadening or flattening at the low frequency end is referred to as Fresnelfiltering when the size of the irregularity exceeds the radius of the fist Fresnel Zone for the wave geometry and it is produced as a result of multiple-scatterings suffered by the radio wave 


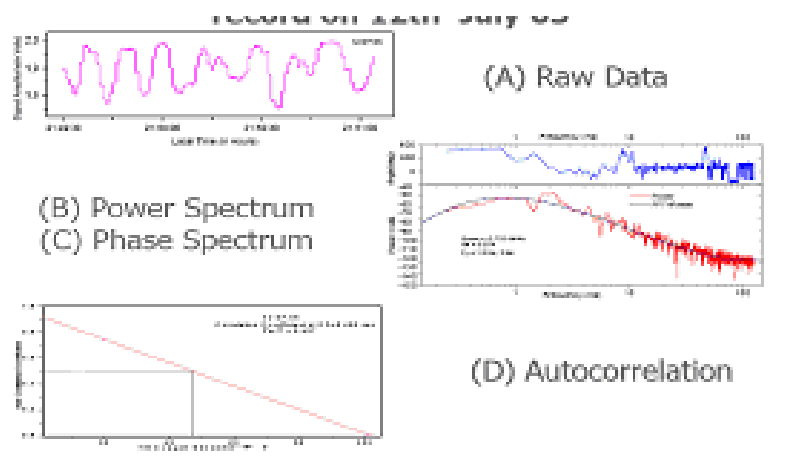

Fig. 3. Power Spectral characteristics of scintillation recorded on 12th July 05, (A) Raw Data, (B)Phase Spectrum, (C) Power Spectrum and (D)Autocorrelation

The usefulness of the spectrum at the low frequency end is determined by the Fresnel-Zone Radius which in turn determines the Fresnel frequency fFR . In the present case the Fresnel frequencies turn out to be $1.5 \mathrm{~Hz}$ and $3.0 \mathrm{~Hz}$. This results in drift velocities of approximately $1500 \mathrm{~m} / \mathrm{sec}$ and $3000 \mathrm{~m} / \mathrm{sec}$ at the rate of $100 \mathrm{~m} / \mathrm{sec}$ for $0.1 \mathrm{~Hz}$, $[8,14,21]$. The second break frequency around $3.0 \mathrm{~Hz}$ in the spectrum indicates that the wave has undergone multiple scatterings with moderately large number of scale sizes of larger irregularities. The high-frequency rolloff is moderately steeper with a slope of $10-2.73 \mathrm{~dB} / \mathrm{Hz}$, indicating a power low nature of the spectrum defined by $\mathrm{Sa}$ $\mathrm{f}-2.73$. This also confirms the moderately strong nature of the scintillation where only a smaller number of large (scale) size irregularities are responsible for causing the scintillations, while the smaller size irregularities are relatively absent during this post-midnight event. Further, an examination of the Fig. 3 (D) shows the "auto-correlation" interval ' $\mathrm{Tm}$ ' is found to be 0.468 seconds while total time period $\mathrm{T}$ is 1.1 seconds. This results in a $\mathrm{Tm} / \mathrm{T}$ ratio of 0.457 that also confirms the moderately strong nature of the scintillation event.

\section{1st January 2006}

This is a scintillation event recorded during winter period, recorded with A/D converter of 256 samples/second and is a postmidnight scintillation event with $S 4=0.458$ which come under a strong category of scintillation. The scintillation record is presented in Fig. 4 (A). The resulting phase spectrum and power spectrum are presented in Fig. $4(\mathrm{~B}, \mathrm{C})$ respectively. The spectral power is expressed in arbitrary units in $\mathrm{dB}$. The "auto-correlation" function is presented in Fig. 4 (D). The power spectrum shown in Fig.. 4 (B) exhibits a slope at the low frequency end followed by a steep roll-off $(-2.557 \mathrm{~dB} / \mathrm{Hz})$ at the high-frequencies showing an asymptotic breaks points around $1.2 \mathrm{~Hz}$ and 6.0 $\mathrm{Hz}$ are the Fresnel-Frequencies [8,14,21] The magnitude of short sloping at the low frequency-end and is referred to as
Fresnelfiltering when the size of the irregularity exceeds the radius of the first Fresnel Zone for the wave geometry and it is produced as a result of multiple scattering. The FresnelZone Radius, which in turn determines the Fresnel frequency, which turn out to be $1.2 \mathrm{~Hz}$ and $6.0 \mathrm{~Hz}$. This results in drift velocities of approximately $1200 \mathrm{~m} / \mathrm{sec}$ and $6000 \mathrm{~m} / \mathrm{Sec}[8,14]$

The high-frequency roll-off is steeper (10-2.557 $\mathrm{dB} / \mathrm{Hz}$ ) indicating a power low nature of the spectrum defined by S a f-2.557 also confirms the strong scintillation activity where very large number of scale size irregularities are responsible for affecting multiple scattering and causing the scintillations during this post-midnight hours. Further, an examination of auto correlation shown in Fig.4 (D) shows the interval ' $\mathrm{Tm}$ ' is found to be 0.48 seconds while total time period $\mathrm{T}$ is 101.86 seconds. This results in a $\mathrm{Tm} / \mathrm{T}$ ratio of 0.470 .

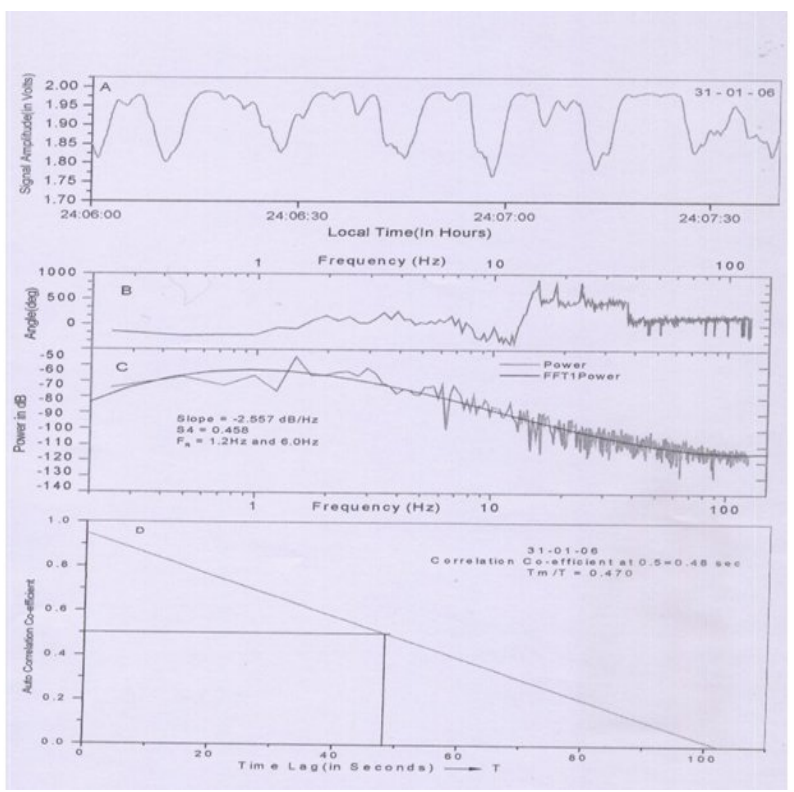

Fig. 4. Power spectral characteristics of scintillation record on 31st January 06 (A) Raw Data (B) Phase Spectrum (C) Power Spectrum (D) Autocorrelation

\section{1th March2006}

This is a scintillation event recorded during equinox season with a high speed $A / D$ converter with a sampling rate of 256 amples/second. Further, this is a premidnight event with S4 $=0.468$ hence, it is characterized as a very strong scintillation. The scintillation record (Raw Data of Amplitude Scintillations) is presented in Fig.5 (A). The resulting phase spectrum and power spectrum are presented in Fig. $5(\mathrm{~B}, \mathrm{C})$ respectively. The spectral power is expressed in arbitrary units in $\mathrm{dB}$. The auto-correlation function is presented in Fig. .5 (D). The power spectrum as shown in Fig.5 (B) exhibits typical characteristics. The very 
low frequency components $<0.5 \mathrm{~Hz}$ are completely absent in this spectrum. The total absence of frequency components below $0.5 \mathrm{~Hz}$ indicates that it is a very strong scintillation process in which very large sized irregularities as well as very large range of irregularity scale sizes are involved in the scattering process [21]. The asymptotic breakpoint is around $2.0 \mathrm{~Hz}$ which is the Fresnel frequency $[8,14,21]$. The usefulness of the spectrum at the low frequency-end is determined by the first Fresnel Zone Radius, which in turn determines the Fresnel frequency.

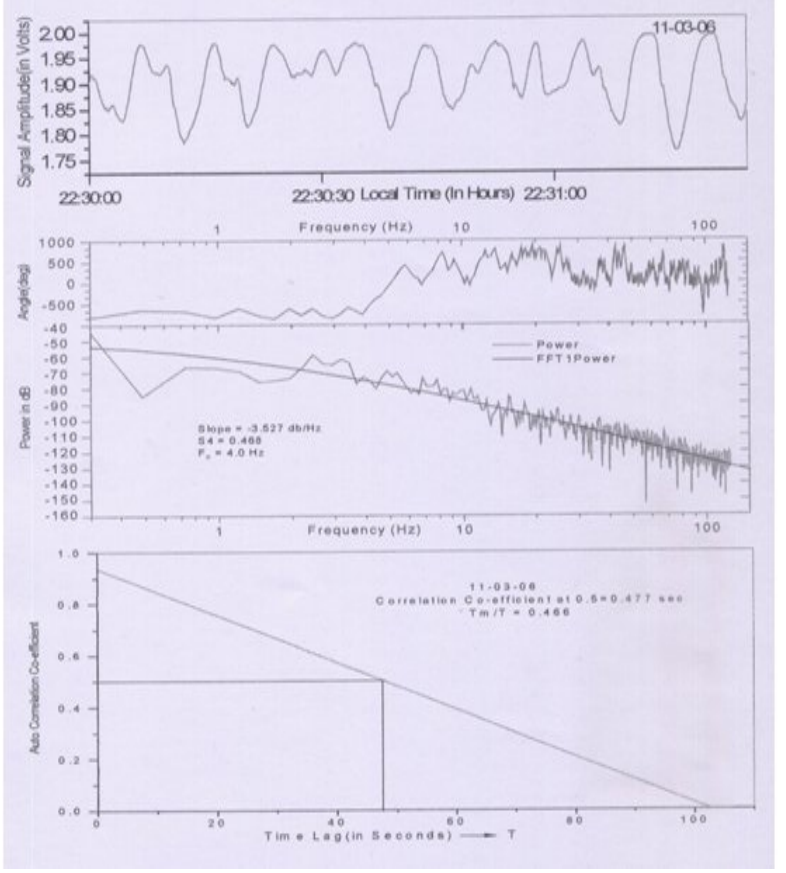

Fig. 5. Power spectral characteristics of scintillation record on 11 March 06 (A) Raw Data (B) Phase Spectrum (C) Power Spectrum (D) Autocorrelation

In the present case which turns out to be $4.0 \mathrm{~Hz}$ in this case. This results in a very high drift velocity of approximately $4000 \mathrm{~m} / \mathrm{sec}$ at the rate of $100 \mathrm{~m} / \mathrm{sec}$. for $0.1 \mathrm{~Hz}$, Fresnel frequency $[8,14,21]$ The high frequency roll-off is steeper between $0.5 \mathrm{~Hz}$ and $2.0 \mathrm{~Hz}$ and slope of the spectrum between $0.4 \mathrm{~Hz}$ and $4.0 \mathrm{~Hz}$ is $10-3.527$ indicating a powerlow nature of the spectrum defined by $\mathrm{S}$ a $\mathrm{f}-3.527$, which also indicates the participation of very large range of scale size irregularities in the scattering process.Further, an examination of the Fig.9 (D), the "autocorrelation-interval $\mathrm{Tm}=0.477$ seconds and the total time period $\mathrm{T}=102.72$ seconds. This results in ( $\mathrm{Tm} / \mathrm{T}$ ) ratio of 0.466 .

\section{4th May 2006}

This is a scintillation event recorded during summer period recorded with highspeed A/D converter having sampling rate of 256 samples/second. Further, this is a premidnight event with S4 $=0.519$ and represents strong scintillation. The scintillation record is presented in Fig.6.(A). The resulting phase spectrum and power spectrum are presented in Fig. 6.(B,C) respectively. The spectral power is expressed in arbitrary units in $\mathrm{dB}$. The "auto-correlation" function is presented in Fig.6 (D). The power spectrum showed in Fig. 6.(D) exhibits typical characteristics. Having irregularities in the scattering process. Further, it also exhibits a steep roll off $(-3.082$ $\mathrm{dB} / \mathrm{Hz}$ ) from $3 \mathrm{Hzto} 15 \mathrm{~Hz}$ The power spectrum also exhibits asymptotic breaks around $0.5 \mathrm{~Hz}$ and around $3 \mathrm{~Hz}$. These break points are known as Fresnel frequencies [8,14,21]. The usefulness of the spectrum at the low frequency end is determined by the Fresnel-Zone Radius, which in turn determines the Fresnel frequency. In the present case which turn out to be $0.5 \mathrm{~Hz}$ and $3.0 \mathrm{~Hz}$. This results in drift velocities of approximately $500 \mathrm{~m} / \mathrm{sec}$ and $3000 \mathrm{~m} / \mathrm{sec}[8,1]$

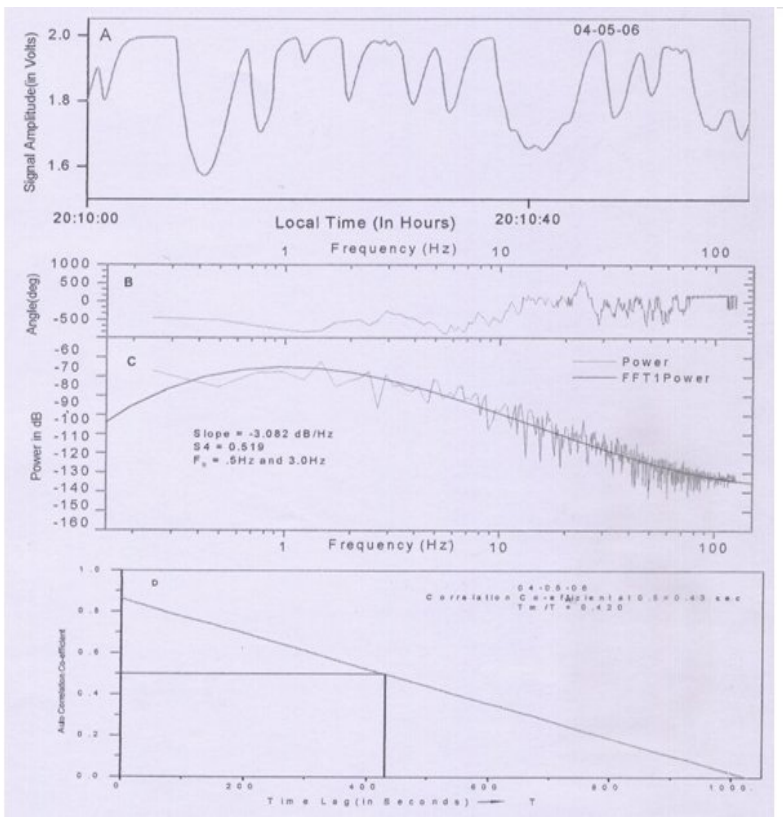

Fig. 6. Power spectral characteristics of scintillation record on 4th May 06 (A) Raw Data (B) Phase Spectrum (C) Power Spectrum (D) Autocorrelation

The high-frequency roll-off within the frequency range of 0.5 and $3.0 \mathrm{~Hz}$ is steeper $(10-3.082 \mathrm{~dB} / \mathrm{Hz})$ indicating a power low nature of the spectrum defined by $S$ a f-3.082 . This also confirms that the scintillation is strong where very large range of scale size irregularities is responsible for causing the scintillations, this pre-midnight event. Further, an examination of the Fig.6. (D) Shows the "autocorrelation" interval ' $\mathrm{Tm}$ ' is found to be 0.43 seconds while total time period $\mathrm{T}$ is 1024.2 seconds. This results in a $\mathrm{Tm} / \mathrm{T}$ ratio of 0.420 . 


\section{STUDY OF POWER SPECTRA DURING TOTAL LUNARECLIPSE ON}

7TH SEPTEMBER 2006.

\section{Power spectral studies of the scintillation recorded on 7th September 2006 - The day preceding the total lunar eclipse day.}

In the present study an attempt has been made by the author to study the influence

of the Solar Eclipse (partial in India) on $11^{\text {th }}$ November 2005 and a complete Lunar Eclipse

on 7th September 2006. It is observed from the data recorded for the eclipse days that there was no any influence of the solar eclipse on the generation or inhibition of Scintillation. But in the case of Lunar eclipse there is a scintillation occurrence on the preceding day of the eclipse and no occurrence on the day of eclipse and the following day. The power spectral studies made for the data recorded on 7th September 2006 using high speed A/D converter with 256 samples/second is a daytime scintillation of very rare phenomena with $\mathrm{S} 4=0.172$ is characterized as a very weak scintillation. The scintillation record (Raw Data of Amplitude Scintillations) is presented in Fig.7.(A). The resulting phase spectrum and power spectrum are presented in Fig.7. (B,C) respectively. The spectral power is expressed in arbitrary units in $\mathrm{dB}$. The "autocorrelation" function is presented in Fig.7 (D).

The power spectrum shown in Fig13 (B) exhibits a flat broadening at the lower frequency up to $1 \mathrm{~Hz}$ with Fresnel frequencies of 0.7 and $1.2 \mathrm{~Hz}$. The high-frequency roll-off is moderately steeper $(10-2.463 \mathrm{~dB} / \mathrm{Hz})$ indicating a power low nature of the spectrum defined by $\mathrm{S}$ a $\mathrm{f}-2.463$. This indicates that the scintillation is a very weak scintillation, where small size of irregularities are responsible for affecting multiple scattering of the radio wave and causing normal scintillations, while the irregularities smaller than the Fresnel zone radius are to be responsible for the modulations superposed over the normal scintillations during this pre-midnight event.

Further, the spectrum exhibits nearly constant high frequency response after $1.0 \mathrm{~Hz}$, which indicates the sudden disappearance of large size irregularities. Fig.7 (D) shows the "autocorrelation" interval 'tm' is found to be 17.84 seconds while total time period $\mathrm{T}$ is 39.46 seconds. This results in a $\mathrm{Tm} / \mathrm{T}$ ratio of 0.452 .

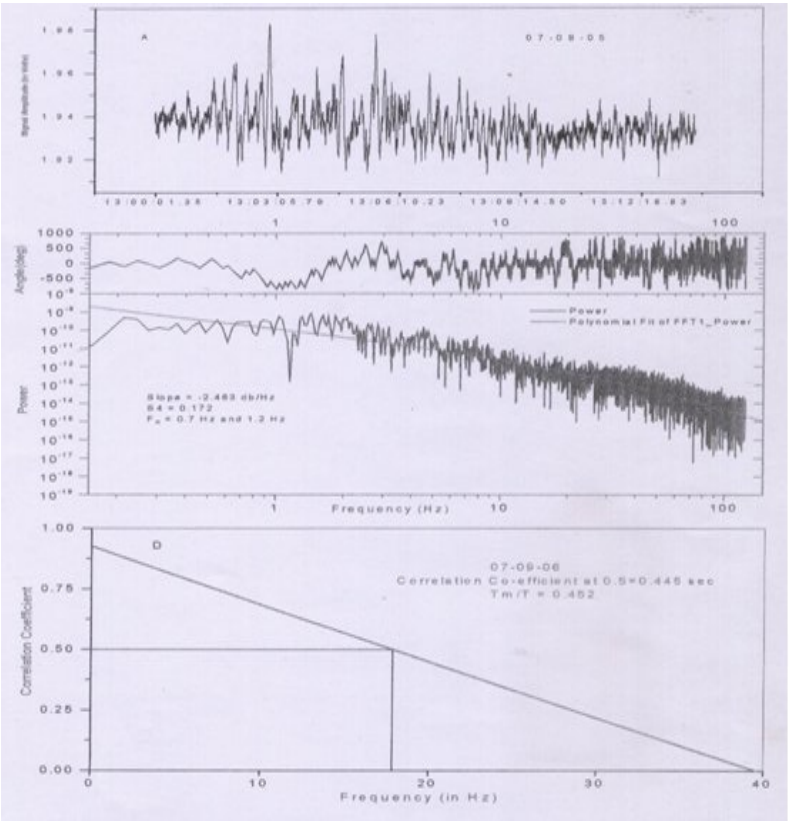

on 7th September 06 (A) Raw Data (B) Phase Spectrum (C) Power Spectrum (D) Autocorrelation

\section{DISTRIBUTION OF THE PERCENTAGE OCCURRENCE OF SPECTRAL INDEX-P}

For a power law type of irregularity (scintillation) spectrum under simple scatter approximation, [13,2] showed the frequency dependence of $\mathrm{S} 4$ as

$$
\text { S4 af }-n \text {.... }
$$

Where $n=(P+2) / 4$. [9] experimentally estimated the value of ' $n$ ' as 1.5 that corresponds to a 'P' value of 4.0. In the present study 200 scintillation records are subjected to power spectral analysis and the corresponding values of $\mathrm{SI}$, $S 4, n, P$ values are computed. The magnitudes of spectral index ' $P$ ' range between a minimum of 2.23 to a maximum of 3.74 with a mean value of 2.98 . The histogram of the frequency distribution of the percentage occurrence of spectral index ' $P$ ' with different magnitudes is presented in Fig.8.. It is observed from the figure that the occurrence of spectral index with a magnitude of -2.7 is found maximum at about $23.53 \%$ followed by a magnitude of -2.5 at about $17.42 \%$ and the spectral indices with magnitudes of -3.0 to 3.7 shows a gradual rapid fall. This indicates that the probability of occurrence of very strong scintillations is in fewer occurrences. That is the moderate scintillations occur more frequently, whereas the probability of occurrence of very weak and very strong scintillations is a rare phenomenon for the period 2005-06. This is a typical characteristic feature of this near-equatorial station, Anantapur as compared to other stations 


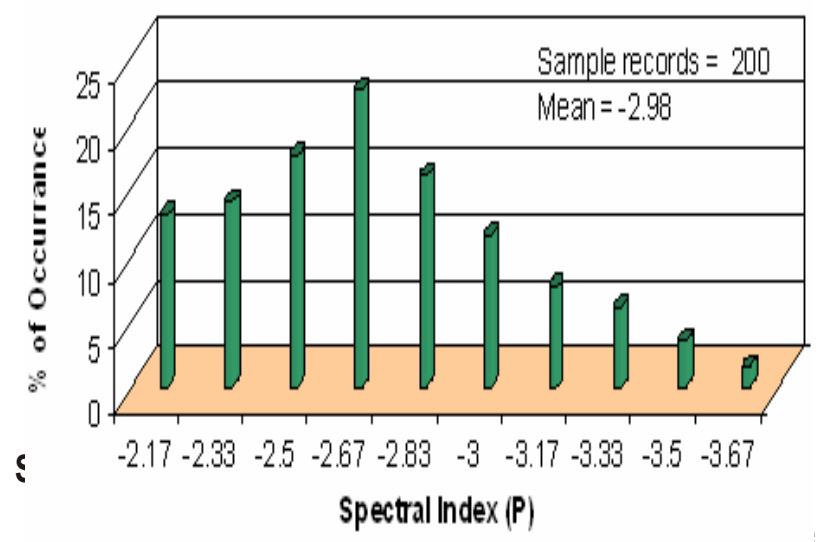

power spectral studies and results of the analysis are presented in Table 1.

\section{Results of the Power Spectral Studies}

The irregular plasma structure being anisotropic in the scattering medium imposes a random phase and intensity fluctuation on the transmitted electromagnetic wave such as

1. The power spectra shows general flatness at low frequencies and high frequency roll off with definite break in the range $0.2 \mathrm{~Hz}$ to $6.0 \mathrm{~Hz}$ indicating the Fresnel-frequency.

2. The high frequency roll off was generally linear in loglog plot indicating power law nature of the spectrum.

3 Auto correlation functions and spectral index S4 shows that the pre-mid night scintillations are fast and postmidnight scintillations are slow.

4. The phase spectrum is related to the power (intensity) spectrum for weak scattering (single scattering) Saltpeter,(1967).

5. Heavy attenuation and severe intensity fluctuations are produced by irregularity structures larger than the Fresnel-Zone Radius.

6. A sinusoidal type modulation of intensity spectrum is produced by irregularity structures smaller than the Fresnel radius.

7. The power spectral study serves as a powerful tool and provide information about the nature of irregularities with scale sizes between first Fresnel zone radius and spatial frequency corresponding to the Nyquist Frequency.

8. In general, the power spectrum of a scintillating signal, shows a flat low frequency response followed by rolloff at high frequencies with an asymptotic break in between. This break indicates the Fresnel frequency The amount of broadening or flattening at the low frequencies is

referred to as Fresnel filtering when the size of the irregularity exceeds the

radius of the first Fresnel Zone for the wave geometry and it is produced as a result of multiple scattering when the size of the irregularity exceeds the radius of the first Fresnel Zone.Further, the Scintillation Index (SI), which indicates the extent of irregularity scale sizes, characterizes the magnitude of broadening but not by S4 Index, which indicates the extent of scintillation suffered by a radio wave.

9. The high frequency roll-off is generally linear in log-log plot indicating the power law nature of the spectrum given by $P \sim f-n$ where $n=(P+2) / 4$ and $P$ is the Power Index

\section{Results of the Auto-Correlation Study and Auto-} Correlation Interval and

\section{$(\mathrm{Tm} / \mathrm{T})$ Ratio}

It is observed from the analysis of the auto correlation functions for the pre-midnight hours (for strong scintillations) is steeper and falls more rapidly than that of the postmidnight scintillations. Hence, the time interval ' $\mathrm{m}$ ' required for the auto-correlation coefficient to reduce to 0.5 is are almost similar during pre-midnight and post-midnight hours(for moderate/weak scintillations).

\section{Power Spectral Slopes}

1. The powers of the spectral slopes are deep shallower in case of pre-midnight scintillations and steeper in case of postmidnight scintillations.. The mean slopes being 2.87 and 2.0 respectively for premidnight and post-midnight scintillations.. According to [21] large range of (irregularity) scale-sizes (meter to kilometer) are involved in the generation phase of the irregularities during pre-midnight hours which are responsible for multiple scatterings, while the absence of small range of scale sizes of irregularities during post-midnight hours is responsible for stronger scintillations.

2. Spectral slopes are steeper for strong scintillations and shallower for weak scintillations.

\section{REFERNECES}

[1] Aarons, J., Whitney, H.E. and Allen, R.S. 1971.Global Morphology of Ionospheric scintillations, Proc. IEEE, 59, 159-172. 
[2] Basu, S. and Whitney, H.E. 1983. The temporal structure of intensity sectors near the magnetic equator. Radio Sci., 18, 263-271.

[3] Basu, S., Basu, Su, Mc Clure, J.P., Hanson, W.D. and Whitney, H.E. 1983. High resolution topside insitu data of electron densities and $\mathrm{VHF} / \mathrm{GHz}$ scintillations in the equatorial regin. 0 . Veophys. Res., 88, 403-415.

[4] Basu, S., Basu, Su., Aarons, J., Mc Clure, J.P. and Cousins, M.D. 1978. On the co-existance of km-m scale irregularities in the nighttime equatorial $\mathrm{F}$ region. 0. Veophys. Res., 83 4219-4226.

[5] Blackman, R.B. and Tukey, J.D. 1958. The Measurement of Power Spectra, Dover Publications, New York.

[6] Bramely, E.N. and Browning, R. 1978. Midlatitude ionospheric scintillation of geostationary satellite signals at $137 \mathrm{MHz}$. 0. Atmos. Terr. Phys., 40, 12471255.

[7] Budden, K.G. 1965. The Amplitude Fluctuations of the Radio Wave Scattered from a Thick lonospheric Layer with Weak Irregularities, 0.Atoms. terr. Phys., 27, 155-172.

[8] Chandra, H., Vyas, G.D., Sinha, H.S.S., Mishra,R.N. and Prakash, S. 1992. Ionospheric scintillation observations from SHAR. 0.Atmos. Terr. Phys., 54, 167-172.

[9] Crane, R.K. 1976. Spectra of Ionospheric Scintillation, to appear in 0. Veophys. Res. [10] Davies, K., fritz, R.B., Grubb, R.N. and Jones, J.E. 1975. ATS-6 radio beacon experiment: The First Year, IEEE Trans. Aerospace and Elect. Sys., AES11, 1103-1107.

[11] Elkins, T.J. and Papaginnis, M.D. 1969.Measurement and Interpretation of Power Spectrums of Ionospheric Scintillation at a Subauroral Location, 0. Veophys. Res. , 74, 4105-4115.

[12] Franke, S.J., Liu, C.H. and Fang, D.J. 1992.Kersley, L. and Chandra, H. 1984. Mullifrequency study of ionospheric scintillation at Asce nsion Island, Radio Sci., 19, 696-706.

[13] Jokippi, J.R. and Hollweg, J.V. 1970.Interplanetary Scintillation and the Structure of Solar-wind fluctuations, Astrophys. 0., 160, 745-753.

[14] Kersley, L. and Chandra, H. 1984. Power spectra of
VHF intensity Scintillations from $\mathrm{F} 2$ and $\mathrm{E}$ region ionospheric irregularities. 0. Atmos. Terr. Phys., 46, 667-672.

[15] Krishnamoorthy, Raghava Reddy, K.C. and Krishnamurthy, B.V. 1979. Nighttime ionospheric scintillations at the magnetic equator. 0. Atmos. Terr. Phys., 41, 123-134.

[16] Liu, C.H. and Yeh, K.C. 1975. Frequency and Spatial Correlation Functions in a Fading Communication Channel through the lonosphere, Radio Sci., 10, 1055-1061.

[17] Liu, C.H., Wernik, A.W., Yeh, K.C. and youakim, M.Y. 1974. Effects of Multiple scattering on Scintillation of Trans-ionospheric radio signals, Radio Sci., 9, 599607.

[18] Lovelace, R.V.E., Salpeter, E.E., and Sharp, L.E. 1970. Analysis of Observations of Interplanetary Scintillations, Astrophys. 0., 159, 1047-1055.

[19] Marians, M. 1975. Computed Scintillation Spectra for Strong Turbulence, Radio Sci., 18, 115-119.

[20] Oppenheim, A.V. and Sehafer, R.W. 1975.Digital Signal Processing, Prentice-Hall Inc., New Jersey.

[21] Pathak, K.N., Mathew, B., Pandey, R. and lyer, K.N. 1992. Power spectral studies of lonospheric Scintillations. Proc. Of International Becoan Satellite Symposium, 170-175.

[22] Prakash, S., Pal, S. and Chandra, H. 1991. In-situ studies of equatorial spread-F over SHAR -Steep gradients in the bottomside F-region and transitional wavelength results. 0. Atmos. Terr. Phys., 53, 977986.

[23] Rino, C.L. and fremouw, E.J. 1973. Statistics for lonospherically Diffracted VHF/UHF signals. Radio Sci., 8, 223-233.

[24] Rufenach, C.L. 1971. A radio scintillation method of estimating the small scale structure in the ionosphere. 0.Atmos. Terr. Phys., 33, 1941-1951.

[25] Rufenach, C.L. 1972. Power-law Wavenumber spectrum Deduced from lonospheric Scintillation Observations, 0. Veophys. Res., 77, 4761-4272.

[26] Rufenach, C.L. 1975. Power Spectra of Large Scintillation Signals, 0. Atmos. Terr. Phys., 37, 569572.

[27] Rumsey, V.H. 1975. Scintillations due to a concentrated Layer with a Power Law Turbulence spectrum, Radio Sci., 19, 107-114. 
[28] Saltpeter, E.E. 1967. Interplanetary scintillation I., TheoryAstrophys. 0., 142, 433-448.

[29] Shishov, V.I. 1971. Diffraction of Waves by a strongly Refracting Random Phase Screen, Radiofizika, 14, 85-92.

[30] Singleton, D.G. 1974. Power spectra of lonospheric Scintillations, 0.Atmos. Terr. Phys., 36, 113-133.

[31] Tatarski, V.I. 1971. 1971. The Effects of the Turbulent Atmosphere on Wave Propagation, U.S. Dept. of Commerce, National Technical Information Service, Springfield, Va.

[32] Wernik, A.W. and Liu, C.H. 1974. Ionospheric Irregularities causing Scintillation of $\mathrm{GHZ}$ Frequency Radio Signals, 0. Atmos. Terr. Phys., 36, 871-879.

[33] Yeh, K..C., Liu, C.H. and Youakim, M.Y. 1975. A Theoretical study of the lonospheric Scintillation Behavior caused by Multiple scattering, Radio Sci., 10, $97-106$

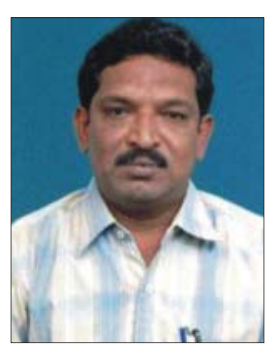

Dr.U.Eranna received his B.Tech, M.E and Phd from JNTU college of Engineering Anantapur, Faculaty of Technology and Engg Baroda and SK University Anantapur respectively. He is an eminent professor and Head of the department of Electronics and Communication Engg at Bellary Engineering College, Bellary, having 20 years of admirable teaching experience and his current research interests are in lonospheric studies. He is also dynamically involved in Administrative activities, academic activities and also Member of the Advisory Committee of the College, since the establishment of the Bellary Engineering College, Bellary, which results to superior status of the college in the Gulbarga Region of VTU within 3 years of its establishment.. 